\title{
Finite Element Analysis of Beams with Nonlocal Foundations
}

\author{
Yongjun Lei* \\ National University of Defense Technology, PR China \\ Michael I. Friswell ${ }^{\dagger}$ and Sondipon Adhikari ${ }^{\ddagger}$ \\ University of Bristol, UK
}

\begin{abstract}
In this paper, a nonlocal viscoelastic foundation model is proposed and used to analyse the dynamics of beams with different boundary conditions using the finite element method. Unlike local foundation models the reaction of the nonlocal model is obtained as weighted average of state variables over a spatial domain via convolution integrals with spatial kernel functions that depend on a distance measure. In the finite element analysis, the interpolating shape functions of the element displacement field are identical to those of standard two-node beam elements. However, for nonlocal elasticity or damping, nodes remote from the element do have an effect on the energy expressions, and hence the damping and stiffness matrices. The expressions of these direct and cross stiffness and damping matrices may be obtained explicitly for some common spatial kernel functions. Alternatively numerical integration may be applied to obtain solutions. Numerical results for eigenvalues and associated eigenmodes of Euler-Bernoulli beams are presented and compared (where possible) with results in literature using exact solutions, Galerkin approximations or the transfer matrix approach. The examples demonstrate that the finite element technique is efficient for the dynamic analysis of beams with nonlocal viscoelastic foundations.
\end{abstract}

\section{Introduction}

Beam and beam-like structures resting on elastic or viscoelastic foundations have wide application in modern engineering practices, such as, railway tracks, highway pavements, and continuously supported pipelines. As a result, numerous studies have been performed to investigate the static deflection, the dynamic response, and the dynamic stability of beams on elastic or viscoelastic foundations. However, in most of these studies, the foundation was idealized as a one-parameter Winkler model, where the system is modelled as infinitely close linear springs and the foundation pressure at any point is proportional to the deflection at that point. Several more complicated models have been reported in the literature, such as the two-parameter Pasternak model, the three-parameter Kerr model, the Vlasov model, or fractional derivative viscoelastic models.

Yankelevsky and Eisenberge ${ }^{1}$ used the analytical expressions for the deflection of a finite beam with unit end loads as interpolating shape functions for a finite element to analyze the static deflection of a beam column on an elastic foundation. Thambiratnam and Zhuge ${ }^{2}$ also used the finite element method to investigate the free vibration of variable thickness thin beams supported on elastic foundations. Sun ${ }^{3}$ presented the closed-form solution for an Euler-Bernoulli beam on a viscoelastic foundation excited by harmonic line loads using a Green's Function approach. Kim ${ }^{4}$ applied the Fourier transform to obtain the solution for the vibration and stability of axial loaded beams on elastic foundations with moving harmonic loads. Other authors analysed the static and dynamic respone of other structural components (rods, plates, shells). ${ }^{5-8}$ In most of these studies, the foundation is idealized as a Winkler (one-parameter) model. In the Winkler model, the foundation is modelled as a system composed of infinitely close linear springs,

\footnotetext{
*Associate Professor, College of Aerospace and Material Engineering.

${ }^{\dagger}$ Sir George White Professor of Aerospace Engineering, Department of Aerospace Engineering, Bristol BS8 1TR.

${ }^{\ddagger}$ Lecturer, Department of Aerospace Engineering, Bristol BS8 1TR.
} 
and assumes that the foundation pressure at any point is proportional to the deflection at that point. The Winkler model does not accurately represent the characteristics of many practical foundations. One of the most important shortcomings of this model is that it assumes no interactions between the springs or discontinuities in the foundation. To overcome these problems, several more complicated models have been suggested in the literature, such as the Pasternak (two-parameter), Kerr (three-parameter), or Vlasov models. For example, Yokoyama ${ }^{9}$ proposed a finite element method to determine the vibration characteristics of a uniform Timoshenko beam-column supported on a two-parameter elastic foundation. Eisenberger ${ }^{10}$ introduced an exact method to compute the natural frequencies of Euler beams on a two-parameter elastic foundation. Chen et al. ${ }^{11}$ combined the state space and differential quadrature methods to develop a mixed method for the bending and free vibration of a beam resting on a Pasternak elastic foundation. Similar problems for uniform beams on elastic foundations were treated by $\mathrm{Chen}^{12,13}$ using the differential quadrature method. de Rosa and Maurizi ${ }^{14}$ investigated the influence of a concentrated mass and the Pasternak soil foundation model on the free vibration of Euler-Bernoulli beams. Avramidis and Morfidis ${ }^{15}$ studied the static bending of Timoshenko beams on Kerr-type, three-parameter elastic foundations. Ayvaz and Ozgan ${ }^{16}$ considered the modified Vlasov model to analyze the free vibration of beams resting on elastic foundations, and analyzed the effects on the natural frequencies of the subsoil depth, the beam length, their ratio and the value of the vertical deformation parameter within the subsoil. Atanackovic and Stankovic ${ }^{17}$ and Fenander ${ }^{18}$ investigated the stability and dynamic response of elastic beams on viscoelastic foundations modelled using fractional derivatives.

All of the papers described above are formulated in terms of local field theory, where the foundation reactions are directly proportional to the state variables (displacement, rotation, curvature) of the beam deflection. For the Winkler-type one-parameter model, the relationship between the foundation reaction $Q_{W}(x, t)$ and the deflection on the foundation surface $w(x, t)$ is $^{2}$

$$
Q_{W}(x, t)=k_{1}(x) w(x, t)
$$

where $k_{1}(x)$ is the stiffness coefficient of the elastic foundation model, and $x$ and $t$ are spatial and time variables, respectively.

For the Pasternak-type two-parameter model, the foundation reaction $Q_{P}(x, t)$ is ${ }^{9}$

$$
Q_{P}(x, t)=k_{1}(x) w(x, t)-G_{1}(x) \frac{\partial w^{2}(x, t)}{\partial x^{2}}
$$

where $k_{1}(x)$ and $G_{1}(x)$ are the stiffness coefficients of the spring and shear layers of the Pasternak foundation model, respectively.

For the Kerr-type three-parameter model, the foundation reaction $Q_{K}(x, t)$ is given by ${ }^{15,19}$

$$
\left(1+\frac{k_{2}(x)}{k_{1}(x)}\right) Q_{K}(x, t)-\frac{G_{1}(x)}{k_{1}(x)} \frac{\partial^{2} Q_{K}(x, t)}{\partial x^{2}}=k_{2}(x) w(x, t)-G_{1}(x) \frac{\partial w^{2}(x, t)}{\partial x^{2}}
$$

where $k_{1}(x), k_{2}(x)$ and $G_{1}(x)$ are the stiffness coefficients of the two spring layers and the middle shear layer for the Kerr foundation model, respectively.

However, Flugge ${ }^{20}$ pointed out that

The reaction $q\left(x_{1}\right)$ at any point $x_{1}$, of course, not only upon the local value $w\left(x_{1}\right)$ of the deflection, but also upon that of neighbour points $x_{2}$, their influence decreasing as the distance $\left|x_{1}-x_{2}\right|$ increases.

Obviously, the Winkler foundation model described by Eq. (1) is a local reaction model and the influence of deflections close to the point of interest have not been considered. Equations (2) and (3) give relatively accurate models for soil foundations compared to the Winkler foundation model. In these models the influence of the deflection at neighbouring points are considered implicitly through the second order derivatives of the deflection with respect to the spatial variable. However these two models are still local models because they do not consider attenuation effects with increasing spatial distance. Hence the motivation to study nonlocal models for elasticity and damping. 


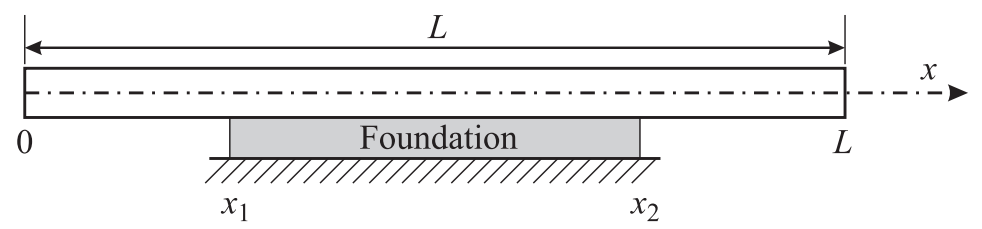

Figure 1. A beam with a partial nonlocal foundation.

\section{The Nonlocal Foundation Model}

In what follows, the elastic Winkler foundation model is generalized to a nonlocal Kelvin viscoelastic foundation model using the nonlocal field theory. ${ }^{21-23}$ Nonlocal elasticity theory has be investigated, however few authors have addressed nonlocal viscoelastic media. ${ }^{24,25}$ In one-dimension nonlocal elasticity, the foundation reaction is given as

$$
Q_{N}(x, t)=\int_{x_{1}}^{x_{2}} K(x, \xi) w(\xi, t) d \xi+\left.\int_{x_{1}}^{x_{2}} \int_{-\infty}^{t} C(x, \xi, t-\tau) \frac{\partial w(\xi, t)}{\partial t}\right|_{t=\tau} d \tau d \xi
$$

where $w$ is the bending deflection of the beam, $K(x, \xi)$ is the elastic spatial kernel, $C(x, \xi, t)$ is the damping kernel, and the spatial integrations are over the length of the foundation, whose extent is denoted by $x_{1}$ and $x_{2}$ in figure 1.

The equation of motion for a non-uniform Euler-Bernoulli beam on a nonlocal viscoelastic foundation between $x_{1}$ and $x_{2}$ is then

$$
\frac{\partial^{2}}{\partial x^{2}}\left(E I(x) \frac{\partial^{2} w(x, t)}{\partial x^{2}}\right)+\rho A(x) \frac{\partial^{2} w(x, t)}{\partial t^{2}}+Q_{N}(x, t)\left[H\left(x-x_{1}\right)-H\left(x-x_{2}\right)\right]=f(x, t)
$$

where $f(x, t)$ is excitation force, $E I(x)$ and $\rho A(x)$ are the bending stiffness and mass per unit length of the beam and $H(\cdot)$ is the Heaviside step function. The beam is initially assumed to be at rest and standard boundary conditions are applied at the two ends.

Equation (5) is an integro-differential equation, and obtaining closed form solutions is difficult. Adhikari et $a l .{ }^{26}$ presented a closed-form solution for beams with nonlocal damping by the transfer function method. Lei et al. ${ }^{27}$ presented approximate solutions using a Galerkin method for uniform beams with typical kernel functions. To treat more complicated problems with variable foundation stiffness, non-uniform section properties or with intermediate supports, a finite element method is developed.

\section{A. Typical Kernel Functions}

In this paper we assume that the damping kernel function $C(x, \xi, t)$ is separable in space and time. Thus the kernel function takes the form

$$
C(x, \xi, t-\tau)=C_{0}\left[H\left(x-x_{1}\right)-H\left(x-x_{2}\right)\right] c(x-\xi) g(t-\tau) .
$$

Physically this model represents nonlocal viscoelastic damping, for example, in a beam with viscoelastic material or an actively controlled piezoelectric patch. Viscous damping has the form $g(t-\tau)=\delta(t-\tau)$, and local damping $c(x-\xi)=\delta(x-\xi)$.

The function $g(t)$ is the relaxation kernel function of the non-viscous element and is often taken as

$$
g(t)=\sum_{i=1}^{m} \frac{g_{\infty i}}{\tau_{i}} e^{-t / \tau_{i}}
$$

where $g_{\infty i}$ and $\tau_{i}$ are positive constants representing the damping coefficients and relaxation times, respectively. The Laplace Transform of the relaxation kernel is then

$$
G(s)=\sum_{i=1}^{m} \frac{g_{\infty i}}{\tau_{i} s+1}
$$




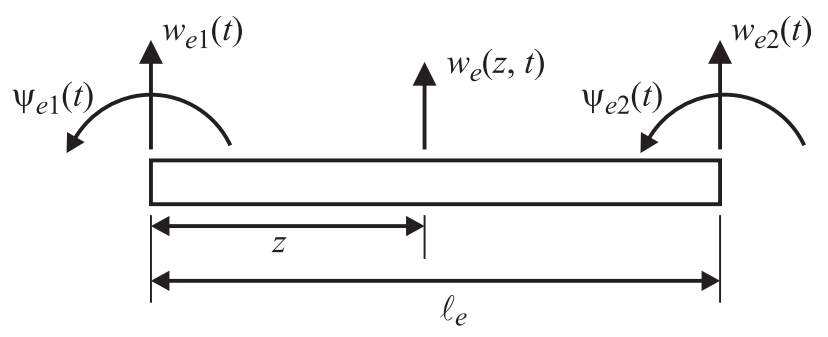

Figure 2. The degrees of freedom of a beam finite element.

If $\tau_{i} \rightarrow 0, \forall i$, then one obtains the standard viscous damping model. Alternatively fractional derivative or GHM models could be used. ${ }^{28-32}$

The spatial kernel function, $c(x-\xi)$ in Eq. (6) is normalised to satisfy the condition,

$$
\int_{-\infty}^{\infty} c(x) d x=1 .
$$

Common choices for this kernel function are the exponential decay

$$
c(x-\xi)=\frac{\alpha}{2} e^{-\alpha|x-\xi|},
$$

or the error function

$$
c(x-\xi)=\frac{\alpha}{\sqrt{2 \pi}} e^{-\alpha^{2}(x-\xi)^{2} / 2} .
$$

Other models are also possible. ${ }^{27}$ If $\alpha \rightarrow \infty$ then one obtains the standard local model.

The elastic spatial kernel function $K(x, \xi, t)$ will be similar, expect that the time dependence is assumed to be negligible. Thus

$$
K(x, \xi)=K_{0}\left[H\left(x-x_{1}\right)-H\left(x-x_{2}\right)\right] k(x-\xi)
$$

where $k(x-\xi)$ has the same form as $c(x-\xi)$.

\section{The Finite Element Model}

Generally the approach adopted when developing models using finite element analysis is to approximate the deformation within an element using nodal values of displacement and rotation. The kinetic and strain energy for each element is then computed and the contributions of each element added together to obtain a global model of the structure. The damping matrix is obtained is a similar way using the dissipation function. One key aspect of finite element analysis is that the energy contributions from each element only depends on the displacements and rotations at the nodes associated with that element. Clearly for nonlocal elasticity this will not be the case, although the form of the exponential kernel function given by Eq. (10) does lead to considerable simplification. This paper only considers a nonlocal foundation, and the development of the mass and stiffness matrices for the beam follows the standard procedure, which will be outlined briefly to establish notation.

\section{A. Shape Function Definition and Beam Elements}

A standard beam element is modelled using two nodes (at the ends of the beam element), and two degrees of freedom per node (translation and rotation), as shown in figure 2. The deformation within the eth element, $w_{e}(z, t)$, is approximated using cubic shape shape functions, for $z \in\left[0, \ell_{e}\right]$, as

$$
w_{e}(z, t)=\mathbf{N}_{e}(z) \mathbf{q}_{e}(t), \quad \text { where } \quad \mathbf{N}_{e}(z)=\left[N_{e 1}(z) N_{e 2}(z) N_{e 3}(z) N_{e 4}(z)\right], \quad \mathbf{q}_{e}(t)=\left\{\begin{array}{l}
w_{e 1}(t) \\
\psi_{e 1}(t) \\
w_{e 2}(t) \\
\psi_{e 2}(t)
\end{array}\right\}
$$


and

$$
\begin{array}{ll}
N_{e 1}(z)=1-3 \frac{z^{2}}{\ell_{e}^{2}}+2 \frac{z^{3}}{\ell_{e}^{3}}, & N_{e 2}(z)=z-2 \frac{z^{2}}{\ell_{e}}+\frac{z^{3}}{\ell_{e}^{2}}, \\
N_{e 3}(z)=3 \frac{z^{2}}{\ell_{e}^{2}}-2 \frac{z^{3}}{\ell_{e}^{3}}, & N_{e 4}(z)=-\frac{z^{2}}{\ell_{e}}+\frac{z^{3}}{\ell_{e}^{2}} .
\end{array}
$$

The kinetic energy, for a beam of length $L$ with $M$ elements, is then approximated as

$$
T=\frac{1}{2} \int_{0}^{L} \rho A(x)\left(\frac{\partial w(x, t)}{\partial t}\right)^{2} d x=\frac{1}{2} \sum_{e=1}^{M} \int_{0}^{\ell_{e}} \rho A\left(x_{e}+z\right)\left(\frac{\partial w_{e}(z, t)}{\partial t}\right)^{2} d z=\frac{1}{2} \sum_{e=1}^{M} \dot{\mathbf{q}}_{e}(t)^{\top} \mathbf{M}_{e} \dot{\mathbf{q}}_{e}(t),
$$

where the eth node, corresponding to node 1 of the eth element, is located at $x_{e}$, and the element mass matrix is

$$
\mathbf{M}_{e}=\int_{0}^{\ell_{e}} \rho A\left(x_{e}+z\right) \mathbf{N}_{e}(z)^{\top} \mathbf{N}_{e}(z) d z
$$

\section{B. Nonlocal Stiffness and Damping}

For nonlocal stiffness the global stiffness matrix is obtained in a similar way using the strain energy. Assume that the foundation covers the whole length of the beam; the case of a partial foundation is an easy extension. Only the contribution from the foundation will be considered here, and this is given by

$$
\begin{aligned}
U_{F} & =\frac{1}{2} \int_{0}^{L} Q_{N}(x, t) w(x, t) d x=\frac{1}{2} \int_{0}^{L} \int_{0}^{L} K(x, \xi) w(\xi, t) w(x, t) d \xi d x \\
& =\frac{1}{2} \sum_{i, j=1}^{M} \mathbf{q}_{i}^{T} \mathbf{K}_{i j} \mathbf{q}_{j}
\end{aligned}
$$

where

$$
\mathbf{K}_{i j}=\int_{0}^{\ell_{j}} \int_{0}^{\ell_{i}} k\left(x_{j}+\hat{x}-x_{i}-\hat{\xi}\right) \mathbf{N}_{i}^{T}(\hat{\xi}) \mathbf{N}_{j}(\hat{x}) d \hat{\xi} d \hat{x}
$$

and $\hat{x}$ and $\hat{\xi}$ are local co-ordinates, for example $x=x_{j}+\hat{x}$.

For nonlocal damping the global damping matrix is obtained in a similar way using the energy dissipation function. The dissipation function is

$$
\begin{aligned}
\mathcal{F}(t) & =\frac{1}{2} \int_{0}^{L}\left\{\int_{0}^{L} \int_{-\infty}^{t} C(x, \xi, t-\tau) \dot{w}(\xi, \tau) d \tau d \xi\right\} \dot{w}(x, t) d x \\
& =\frac{1}{2} \int_{-\infty}^{t} g_{e}(t-\tau) \sum_{i, j=1}^{M} \dot{\mathbf{q}}_{i}(t)^{T} \mathbf{C}_{i j} \dot{\mathbf{q}}_{j}(\tau) d \tau
\end{aligned}
$$

where

$$
\mathbf{C}_{i j}=\int_{0}^{\ell_{j}} \int_{0}^{\ell_{i}} c\left(x_{j}+\hat{x}-x_{i}-\hat{\xi}\right) \mathbf{N}_{i}^{T}(\hat{\xi}) \mathbf{N}_{j}(\hat{x}) d \hat{\xi} d \hat{x}
$$

In general the degrees of freedom at all nodes will be coupled within a region where the structure has nonlocal damping behaviour. This means the global damping matrix will be full, and each term must be determined independently. The problems simplifies significantly if the beams are modelled using beam elements of equal length, so that $\mathbf{N}_{i}=\mathbf{N}, \ell_{i}=\ell, \forall i$. Because the kernel function only involves $x_{j}-x_{i}$ the matrices $\mathbf{C}_{i j}$ are equal for a fixed $j-i$. Thus if the foundation covers $M$ elements, only $M$ of the element matrices $\mathbf{C}_{i j}$ or $\mathbf{K}_{i j}$ have to be calculated. Figure 3 illustrates this for a foundation modelled with three elements, which are defined using four nodes (or eight degrees of freedom). Each $4 \times 4$ block matrix in the $8 \times 8$ global matrix is denoted by a shaded square and for each term these block matrices are identical. This idea is developed further for the exponential kernel below, where further simplification is possible. 


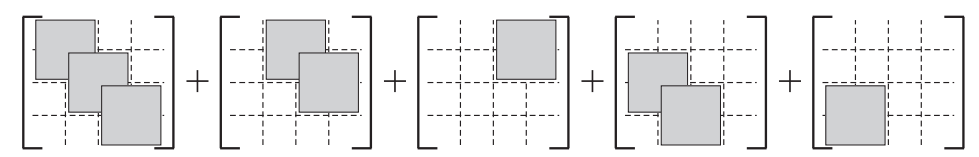

Figure 3. Illustration of the assembly of the global matrices for the nonlocal foundation. For each term depicted by the global matrix, each submatrix denoted by the shaded square is equal.

\section{The Expontential Kernel}

The exponential kernel function given in Eq. (10) leads to a significant simplification in the stiffness and damping matrices, and only requires the calculation of two element matrices. When $i=j$, the direct stiffness matrix is

$$
\mathbf{K}_{i i}=\frac{K_{0} \alpha}{2} \int_{0}^{\ell} \int_{0}^{\ell} e^{-\alpha|\hat{x}-\hat{\xi}|} \mathbf{N}^{T}(\hat{\xi}) \mathbf{N}(\hat{x}) d \hat{\xi} d \hat{x}
$$

If $j>i$

$$
\mathbf{K}_{i j}=\frac{K_{0} \alpha}{2} \int_{0}^{\ell} \int_{0}^{\ell} e^{-\alpha\left(x_{j}-x_{i}+\hat{x}-\hat{\xi}\right)} \mathbf{N}^{T}(\hat{\xi}) \mathbf{N}(\hat{x}) d \hat{\xi} d \hat{x}=e^{-\alpha\left(x_{j}-x_{i}\right)} \overline{\mathbf{K}}=e^{-\alpha(j-i) \ell} \overline{\mathbf{K}},
$$

where the cross stiffness matrix is

$$
\overline{\mathbf{K}}=\frac{K_{0} \alpha}{2} \int_{0}^{\ell} \int_{0}^{\ell} e^{-\alpha(\hat{x}-\hat{\xi})} \mathbf{N}^{T}(\hat{\xi}) \mathbf{N}(\hat{x}) d \hat{\xi} d \hat{x}=\frac{K_{0} \alpha}{2}\left(\int_{0}^{\ell} e^{\alpha \hat{\xi}} \mathbf{N}^{T}(\hat{\xi}) d \hat{\xi}\right)\left(\int_{0}^{\ell} e^{-\alpha \hat{x}} \mathbf{N}(\hat{x}) d \hat{x}\right) .
$$

Similarly if $j<i$,

$$
\mathbf{K}_{i j}=e^{-\alpha(i-j) \ell} \overline{\mathbf{K}}^{T} .
$$

Thus only two element damping matrices need to be computed and then the complete global damping matrix is easily derived. These matrices may be obtained explicitly by integrating Eqs. (21) or (23). Alternatively they may be derived by numerical integration.

As an example to demonstrate how the stiffness matrix is assembled, suppose that a uniform beam is modelled, where three elements have a nonlocal foundation. Then the stiffness matrix will be zero apart from the eight degrees of freedom associated with the foundation. Figure 4 illustrates the assembly and shows the non-zero terms. The global damping matrix for the nonlocal foundation may be assembled in a similar way.

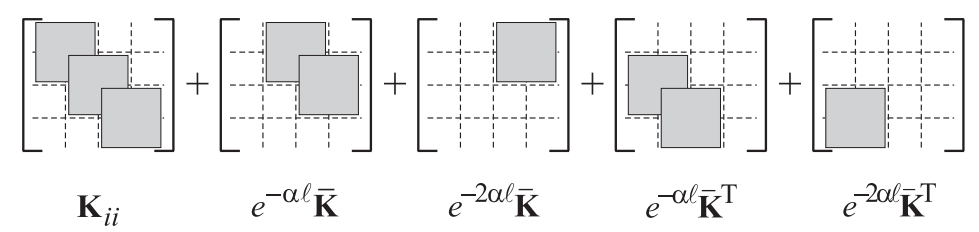

Figure 4. Illustration of the assembly of the global matrices for the nonlocal foundation with an exponential kernel.

\section{The Eigenvalue Problem}

Taking the Laplace transform gives the equations of motion in the form

$$
\left[s^{2} \mathbf{M}+s G(s) \mathbf{C}+\mathbf{K}\right] \mathbf{q}=0
$$

where $\mathbf{M}, \mathbf{K}$, and $\mathbf{C}$ are the global mass, stiffness and damping matrices with respect to the generalised co-ordinates of the beam model. Remember that the stiffness matrix has a contribution from the foundation

$$
6 \text { of } 11
$$


as well as the beam itself. In Eq. (25) the time response of the viscoelastic foundation has been assumed to arise from a single material, leading to a single $G(s)$ term. If different materials are present a damping term will appear for each different relaxation kernel, $G(s)$. Also in Eq. (25), all of the damping is assumed to arise in the foundation; internal damping within the beam is easily incorporated by introducing extra terms.

The eigenvalues are obtained by solving

$$
\operatorname{det}\left|s^{2} \mathbf{M}+s G(s) \mathbf{C}+\mathbf{K}\right|=0 .
$$

The corresponding mode shape functions are obtained by substituting the eigenvalues into Eq. (25) and computing the null space of the matrix. Alternatively, for kernel functions where $G(s)$ is a rational polynomial, the internal variable approach (for example the GHM method ${ }^{30-32}$ ) may be used to produce a linear eigenvalue problem, where the size of the matrices is larger than those in Eq. (26).

\section{Examples}

A number of examples are given to demonstrate the approach. Where possible the finite element solutions are compared to other analyical or approximate methods. Adhikari et al. ${ }^{26}$ described the transfer function method and Lei et $a .^{27}$ described the Galerkin method.

\section{A. A Full Elastic Foundation}

A simply supported uniform beam of length $L=6.096 \mathrm{~m}$ rests on a uniform nonlocal elastic foundation is considered, as shown in figure 1 with $x_{1}=0$ and $x_{2}=L$ (that is the foundation supports the whole beam). This example was also analyzed by Lai et $a l .{ }^{33}$ and Thambiratnam and Zhuge. ${ }^{2}$ The beam has Young's modulus $24.82 \mathrm{GPa}$, second moment of area $1.439 \times 10^{-3} \mathrm{~m}^{4}$ and mass per unit length $446.3 \mathrm{~kg} / \mathrm{m}$. The stiffness of the foundation is $16.55 \mathrm{MNm}^{-2}$. Using the finite element method developed in this paper, the first four natural frequencies of free vibration were obtained with 6,8 and 10 elements, for different values of $\alpha$ for the exponential kernel, Eq. (10). The results are shown in table 1 together with those from the analytical solution, ${ }^{34}$ given by

$$
\omega_{i}=\sqrt{\frac{E I}{\rho A}} \sqrt{\frac{i^{4} \pi^{4}}{L^{4}}+\frac{K_{0}}{E I}} .
$$

Figure 5 shows the effect of varying $\alpha$ on these natural frequencies for the finite element model with 10 elements.

Table 1. Natural frequencies $(\mathrm{Hz})$ of vibration of a simple beam on a nonlocal elastic foundation with an exponential kernel.

\begin{tabular}{ccccccccc} 
& & $\alpha=2$ & & $\alpha=5$ & $\alpha=10$ & $\alpha=50$ & \multicolumn{2}{c}{$\alpha=\infty$} \\
& $N_{e}=6$ & $N_{e}=8$ & $N_{e}=10$ & $N_{e}=10$ & $N_{e}=10$ & $N_{e}=10$ & $N_{e}=10$ & Analytical \\
\hline$\omega_{1}$ & 32.137 & 32.137 & 32.137 & 32.758 & 32.862 & 32.897 & 32.898 & 32.898 \\
$\omega_{2}$ & 55.310 & 55.287 & 55.281 & 56.495 & 56.728 & 56.808 & 56.812 & 56.808 \\
$\omega_{3}$ & 110.89 & 110.62 & 110.54 & 111.61 & 111.86 & 111.95 & 111.95 & 111.90 \\
$\omega_{4}$ & 194.85 & 193.36 & 192.92 & 193.74 & 193.98 & 194.07 & 194.08 & 193.76
\end{tabular}

Suppose now that the kernel consists of the error function, Eq. (11). Table 2 shows the first four natural frequencies for the finite element method with 10 elements. Interestingly the amplitude of $\alpha$ required for the error function kernel to approximate the Dirac delta function (local elasticity) is much higher than that required for the exponential kernel. This is because of the shape of the kernels close to the centre point, as shown in figure 6 .

Table 3 shows the effect of a viscously damped foundation with an exponential kernel for various values of $\alpha\left(K_{0}=0, C_{0}=1 \mathrm{kNsm}^{-2}\right)$. The beam is modelled with 10 finite elements. 


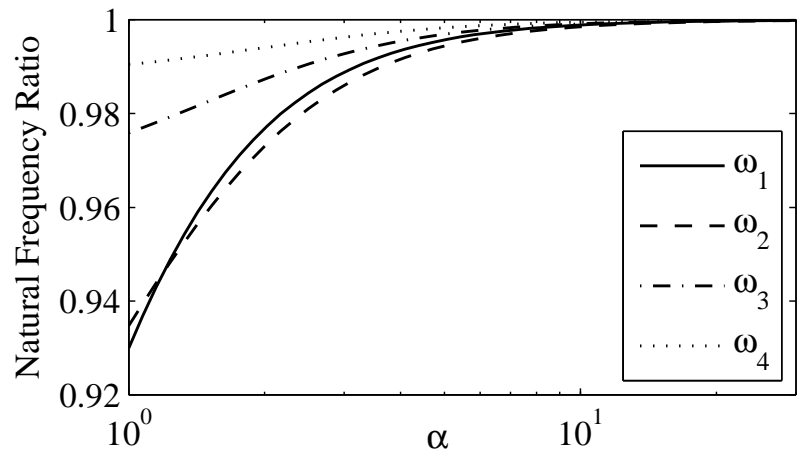

Figure 5. The variation of the first four natural frequencies with $\alpha$ for the fully supported beam. The natural frequencies have been normalised using the values at $\alpha=\infty$.

Table 2. Natural frequencies $(\mathrm{Hz})$ of vibration of a simple beam, modelled with 10 finite elements, on a nonlocal elastic foundation with an error function kernel.

\begin{tabular}{cccccc} 
& $\alpha=5$ & $\alpha=10$ & $\alpha=20$ & $\alpha=50$ & $\alpha=\infty$ \\
\hline$\omega_{1}$ & 42.867 & 38.556 & 35.926 & 32.522 & 32.898 \\
$\omega_{2}$ & 54.724 & 56.013 & 56.476 & 56.593 & 56.812 \\
$\omega_{3}$ & 111.39 & 111.77 & 111.89 & 111.84 & 111.95 \\
$\omega_{4}$ & 193.40 & 193.82 & 193.97 & 194.01 & 194.08
\end{tabular}

\section{B. A Partial Viscoelastic Foundation}

A pinned-pinned beam will be used as an example to demonstrate the use of the finite element methods for a partial nonlocal viscoelastic foundation (that is the foundation stiffness is zero, $K_{0}=0$ ). The results will also be compared to those from the Galerkin method. The $i$ th mode of the undamped beam is $\phi_{i}(x)=$ $A_{i} \sin (i \pi x / L)$ where $A_{i}$ is calculated to give mass normalised mode shapes. These undamped mode shapes will be used as the admissible functions in the Galerkin approach. The dimensions are (see figure 1) $L=$ $200 \mathrm{~mm}, x_{1}=50 \mathrm{~mm}$, and $x_{2}=150 \mathrm{~mm}$. The Young's modulus is $E=70 \mathrm{GN} / \mathrm{m}^{2}$, the density is $\rho=$ $2700 \mathrm{~kg} / \mathrm{m}^{3}$, and the cross-section is $5 \times 5 \mathrm{~mm}$.

Table 4 shows the first 3 eigenvalue pairs for the pinned-pinned beam example with viscous damping and an expontial spatial kernel $(\mu \rightarrow \infty), \alpha=1, g_{\infty}=1$ and $C_{0}=200$, for the Galerkin method with 10

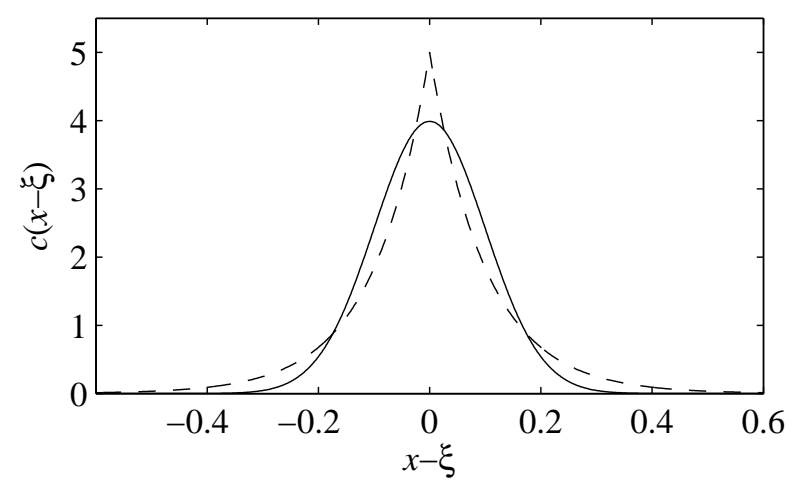

Figure 6. The exponential kernel (dashed), Eq. (10), and error function kernel (solid), Eq. (11), for $\alpha=10$. 
Table 3. Eigenvalues of a simple beam, modelled with 10 finite elements, on a nonlocal viscously damped foundation with an exponential function kernel with $C_{0}=1 \mathrm{kNsm}^{-2}$.

\begin{tabular}{ccccc} 
& $\alpha=2$ & $\alpha=10$ & $\alpha=\infty$ & analytical \\
\hline$\omega_{1}$ & $-1.0613 \pm 75.125 \mathrm{j}$ & $-1.1175 \pm 75.125 \mathrm{j}$ & $-1.1203 \pm 75.125 \mathrm{j}$ & $-1.1203 \pm 75.124 \mathrm{j}$ \\
$\omega_{2}$ & $-0.9157 \pm 300.561 \mathrm{j}$ & $-1.1089 \pm 300.560 \mathrm{j}$ & $-1.1203 \pm 300.560 \mathrm{j}$ & $-1.1203 \pm 300.528 \mathrm{j}$ \\
$\omega_{3}$ & $-0.7443 \pm 676.553 \mathrm{j}$ & $-1.0950 \pm 676.553 \mathrm{j}$ & $-1.1203 \pm 676.553 \mathrm{j}$ & $-1.1203 \pm 676.191 \mathrm{j}$ \\
$\omega_{4}$ & $-0.5891 \pm 1204.11 \mathrm{j}$ & $-1.0761 \pm 1204.11 \mathrm{j}$ & $-1.1203 \pm 1204.11 \mathrm{j}$ & $-1.1203 \pm 1202.12 \mathrm{j}$
\end{tabular}

admissible functions and also for the finite element method with 4, 8 and 40 elements.

Table 5 shows the first 3 eigenvalue pairs for the pinned-pinned beam example with a variety of nonviscous and nonlocal damping, that is as $\mu$ and $\alpha$ vary, for the finite element method with 8 elements.

Suppose now that the beam is clamped at one end and free at the other. All other dimensions are identical to the pinned-pinned beam example. Table 6 shows the first 3 eigenvalue pairs for this cantilever beam example with a variety of non-viscous and nonlocal damping, that is as $\mu$ and $\alpha$ vary, for the finite element method with 8 elements. Clearly both the nonlocal and non-viscous properties of the beam have a significant effect on the damping within the structure.

Table 4. The first 3 eigenvalue pairs for the pinned-pinned beam example obtain from the Galerkin method with 10 admissible functions and the finite element (FE) method with 4,8 and 40 elements. $\alpha=1, g_{\infty}=1$, $C_{0}=200, \mu \rightarrow \infty$

\begin{tabular}{cccc} 
Galerkin & FE (4 elements $)$ & FE (8 elements $)$ & FE (40 elements) \\
\hline$-58.176 \pm 1812.4 \mathrm{j}$ & $-58.174 \pm 1812.9 \mathrm{j}$ & $-58.176 \pm 1812.5 \mathrm{j}$ & $-58.176 \pm 1812.4 \mathrm{j}$ \\
$-0.72086 \pm 7253.5 \mathrm{j}$ & $-0.72080 \pm 7282.1 \mathrm{j}$ & $-0.72086 \pm 7255.4 \mathrm{j}$ & $-0.72086 \pm 7253.5 \mathrm{j}$ \\
$-6.7383 \pm 16320 \mathrm{j}$ & $-6.5458 \pm 16618 \mathrm{j}$ & $-6.7359 \pm 16341 \mathrm{j}$ & $-6.7384 \pm 16320 \mathrm{j}$
\end{tabular}

Table 5. The first 3 eigenvalue pairs for the pinned-pinned beam example obtained from the finite element method with 8 elements for different values of $\mu$ and $\alpha$.

\begin{tabular}{ccccc}
$\mu$ & $\infty$ & 1000 & 1000 & $\infty$ \\
\hline$\alpha$ & 1 & 1 & 10 & 10 \\
\hline & $-58.176 \pm 1812.5 \mathrm{j}$ & $-13.366 \pm 1838.1 \mathrm{j}$ & $-92.216 \pm 2006.0 \mathrm{j}$ & $-447.62 \pm 1757.7 \mathrm{j}$ \\
& $-0.72086 \pm 7255.4 \mathrm{j}$ & $-0.013438 \pm 7255.5 \mathrm{j}$ & $-0.94877 \pm 7262.3 \mathrm{j}$ & $-50.996 \pm 7255.2 \mathrm{j}$ \\
& $-6.7359 \pm 16341 \mathrm{j}$ & $-0.025152 \pm 16342 \mathrm{j}$ & $-0.26562 \pm 16346 \mathrm{j}$ & $-70.624 \pm 16338 \mathrm{j}$
\end{tabular}

\section{Conclusion}

In this paper, a new method of analysing beams with a nonlocal foundation was proposed based on the finite element method. The nonlocal foundation model is a generalisation of the Kevin-Voigt viscoelastic foundation model, where the foundation reaction at a given point depends on the time history and the velocities within a spatial domain. The finite element models for an exponential foundation, for either the stiffness or damping matrices, requires only two matrices, one for the cross element terms and one for the direct terms. The cross matrix to model nonlocal effects is a novel concept introduced in this paper, and these matrices are zero for local damping and elasticity models. Numerical solutions have been obtained for beams with a variety of boundary conditions and foundations. It has been demonstrated that the form of the nonlocal foundation model has a significant impact on the dynamic characteristics of structures. 
Table 6. The first 3 eigenvalue pairs for the cantilever beam example obtained from the finite element method with 8 elements for different values of $\mu$ and $\alpha$.

\begin{tabular}{ccccc}
$\mu$ & $\infty$ & 1000 & 1000 & $\infty$ \\
\hline$\alpha$ & 1 & 1 & 10 & 10 \\
\hline & $-17.841 \pm 645.83 \mathrm{j}$ & $-12.672 \pm 654.28 \mathrm{j}$ & $-102.77 \pm 721.46 \mathrm{j}$ & $-141.58 \pm 634.22 \mathrm{j}$ \\
& $-45.254 \pm 4048.1 \mathrm{j}$ & $-2.6009 \pm 4059.3 \mathrm{j}$ & $-20.277 \pm 4132.1 \mathrm{j}$ & $-353.66 \pm 4009.6 \mathrm{j}$ \\
& $-1.0206 \pm 11343 \mathrm{j}$ & $-0.007875 \pm 11343 \mathrm{j}$ & $-0.47410 \pm 11348 \mathrm{j}$ & $-61.492 \pm 11342 \mathrm{j}$
\end{tabular}

\section{Acknowledgments}

Yongjun Lei gratefully acknowledges the support of China Scholarship Council through a Scholarship Fund Award. Michael Friswell gratefully acknowledges the support of the Royal Society through a Royal Society-Wolfson Research Merit Award. Sondipon Adhikari gratefully acknowledges the support of the Engineering and Physical Sciences Research Council through the award of an Advanced Research Fellowship.

\section{References}

${ }^{1}$ Yankelevsky, D. Z. and Eisenberger, M., "Analysis of beam column on elastic foundation," Computers and Structures, Vol. 23, 1986, pp. 351-356.

${ }^{2}$ Thambiratnam, D. and Zhuge, Y., "Free vibration analysis of beams on elastic foundation," Computers and Structures, Vol. 60, 1996, pp. 971-980.

${ }^{3}$ Sun, L., "A closed form solution of a Bernoulli-Euler beam on a viscoelastic foundation under harmonic line loads," Journal of Sound and Vibration, Vol. 242, 2001, pp. 619-627.

${ }^{4}$ Kim, S.-M., "Vibration and stability of axial loaded beams on elastic foundation under moving harmonic loads," Engineering Structures, Vol. 26, 2004, pp. 95-105.

${ }^{5}$ Fryba, L., Vibration of Solids and Structures under Moving Loads, Thomas Telford, London, 3rd ed., 1999.

${ }^{6}$ de Rosa, M. A. and Maurizi, M. J., "Three approaches for the axial vibrations of bars on modified Winkler soil with nonclassical boundary conditions," Journal of Sound and Vibration, Vol. 231, 2000, pp. 1257-1269.

${ }^{7} \mathrm{Kim}$, S.-M., "Buckling and vibration of a plate on elastic foundation subjected to in-plane compression and moving loads," International Journal of Solids and Structures, Vol. 41, 2004, pp. 5647-5661.

${ }^{8}$ Paliwal, D. N., Pandey, R. K., and Nath, T., "Free vibrations of circular cylindrical shell on Winkler and Pasternak foundations," International Journal of Pressure Vessels and Piping, Vol. 69, 1996, pp. 79-89.

${ }^{9}$ Yokoyama, T., "Vibration analysis of Timoshenko beam-columns on two-parameter elastic foundations," Computers and Structures, Vol. 61, 1996, pp. 995-1007.

${ }^{10}$ Eisenberger, M., "Vibration frequencies for beams on variable one- and two-parameter elastic foundations," Journal of Sound and Vibration, Vol. 176, 1994, pp. 577-584.

${ }^{11}$ Chen, W. Q., Lu, C. F., and Bian, Z. G., "A mixed method for bending and free vibration of beams resting on a Pasternak elastic foundation," Applied Mathematical Modelling, Vol. 28, 2004, pp. 877-890.

${ }^{12}$ Chen, C. N., "Solution of beam on elastic foundation by DQEM," Journal Engineering Mechanics, Vol. 124, 1998, pp. 1381-1384.

${ }^{13}$ Chen, C. N., "Vibration of prismatic beam on an elastic foundation by the differential quadrature element method," Computers and Structures, Vol. 77, 2000, pp. 1-9.

${ }^{14}$ de Rosa, M. A. and Maurizi, M. J., "The influence of concentrated masses and Pasternak soil on the free vibration of Euler beams - exact solution," Journal of Sound and Vibration, Vol. 212, 1998, pp. 573-581.

${ }^{15}$ Avramidis, I. E. and Morfidis, K., "Bending of beams on three-parameter elastic foundation," International Journal of Solids and Structures, Vol. 43, 2006, pp. 357-375.

${ }^{16}$ Ayvaz, Y. and Ozqan, K., "Application of modified Vlasov model to free vibration analysis of beams resting on elastic foundations," Journal of Sound and Vibration, Vol. 255, 2002, pp. 111-127.

${ }^{17}$ Atanackovic, T. M. and Stankovic, B., "Stability of an elastic rod on a fractional derivative type of foundation," Journal of Sound and Vibration, Vol. 277, 2004, pp. 149-161.

${ }^{18}$ Fenander, A., "A fractional derivative railpad model included in a railway track model," Journal of Sound and Vibration, Vol. 212, 1998, pp. 889-903.

${ }^{19}$ Kerr, A. D., "A study of a new foundation model," Acta Mechanica, Vol. 1, 1965, pp. 135-147.

${ }^{20}$ Flugge, W., Viscoelasticity, Springer-Verlag, Berlin, 2nd ed., 1975.

${ }^{21}$ Polizzotto, C., "Non-local elasticity and related variational principles," International Journal of Solids and Structures, Vol. 38, 2001, pp. 7359-7380.

${ }^{22}$ Eringen, A. C., "Theory of nonlocal elasticity and some applications," Res. Mechanica, Vol. 21, 1987, pp. 313-342.

${ }^{23}$ Pisno, A. A. and Fuschi, P., "Closed form solution for non-local elastics bar in tension," International Journal of Solids and Structures, Vol. 40, 2003, pp. 13-23. 
${ }^{24}$ Nowinski, J. L., "On the non-local aspects of stress in a viscoelastic medium," International Journal of Non-Linear Mechanics, Vol. 21, 1986, pp. 439-446.

${ }^{25}$ Ahmadi, G., "Linear theory of nonlocal viscoelasticity," International Journal of Non-Linear Mechanics, Vol. 10, 1975, pp. 253-258.

${ }^{26}$ Adhikari, S., Lei, Y., and Friswell, M. I., "Modal analysis of non-viscously damped beams," Journal of Applied Mechanics, 2006, pp. submitted.

${ }^{27}$ Lei, Y., Friswell, M. I., and Adhikari, S., "A Galerkin Method for Distributed Systems with Non-local Damping," International Journal of Solids and Structures, Vol. 43, 2006, pp. in press.

${ }^{28}$ Bagley, R. L. and Torvik, P. J., "Fractional calculus in the transient analysis of viscoelastically damped structures," AIAA Journal, Vol. 23, 1985, pp. 918-925.

${ }^{29} \mathrm{Kim}$, T.-W. and Kim, J.-H., "Eigensensitivity based optimal distribution of a viscoelastic damping layer for a flexible beam," Journal of Sound and Vibration, Vol. 273, 2004, pp. 201-218.

${ }^{30}$ Golla, D. F. and Hughes, P. C., "Dynamics of viscoelastic structures - a time domain finite element formulation," Transactions of ASME, Journal of Applied Mechanics, Vol. 52, December 1985, pp. 897-906.

${ }^{31}$ McTavish, D. J. and Hughes, P. C., "Modeling of linear viscoelastic space structures," Transactions of ASME, Journal of Vibration and Acoustics, Vol. 115, January 1993, pp. 103-110.

${ }^{32}$ Friswell, M. I., Inman, D. J., and Lam, M. J., "On the Realisation of GHM Models in Viscoelasticity," Journal of Intelligent Material Systems and Structures, Vol. 8, No. 11, November 1997, pp. 986-993.

${ }^{33}$ Lai, Y. C., Ting, B. Y., Lee, W. S., and Becker, W. R., "Dynamic response of beams on elastic foundation," Journal of Structural Engineering, Vol. 118, 1992, pp. 853-858.

${ }^{34}$ Timoshenko, S., Young, D. H., and Weaver, W., Vibration Problems in Engineering, John Wiley and Sons, New York, 4th ed., 1974. 